\title{
Cenpestao
}

\section{Cenários da dinâmica de hipercrescimento e colapso das revistas científicas brasileiras líderes na Ciência da Informação}

\author{
Vinícius Medina Kern \\ Doutor; Universidade Federal de Santa Catarina, Florianópolis, SC, Brasil; \\ v.m.kern@ufsc.br \\ Mauricio Uriona-Maldonado \\ Doutor; Universidade Federal de Santa Catarina, Florianópolis, SC, Brasil; \\ m.uriona@ufsc.br
}

\begin{abstract}
Resumo: Os periódicos brasileiros em Ciência da Informação dependem tipicamente de trabalho semivoluntário, com escassa dotação de recursos. A inclusão de alguns deles em bases de dados importantes lhes confere status e faz atrair mais e mais submissões, sem adequar os recursos disponíveis. Este artigo explora esse contexto por meio de um modelo de dinâmica de sistemas que representa estoques e fluxos de artigos entre a submissão e a decisão editorial final. Simulamos 22 cenários alternativos, com oferta distinta de submissões, com ou sem intervenções (moratória de submissões e aumento agudo de rejeições preliminares). Diversos desses cenários indicam o risco iminente de colapso da revista, independentemente de intervenções que aliviam a sobrecarga apenas momentaneamente. Poucos editores sobrecarregados sustentam a continuidade do sistema.
\end{abstract}

Palavras-chave: Publicações científicas: periódicos. Qualis Capes. Produtivismo acadêmico. Revisão por pares. Comunicação científica.

\section{Introdução}

Os periódicos científicos brasileiros ativos em Ciência da Informação, reunidos em <www.brapci.inf.br>, têm publicação típica em acesso aberto, por um programa de pós-graduação e sem quaisquer taxas. Os recursos essenciais incluem os artigos originais submetidos, o serviço semivoluntário de revisores e editores e um software de gestão editorial (frequentemente o gratuito Open Journal Systems ${ }^{l}$, ou a sua tradução, Sistema Eletrônico de Editoração de Revistas $^{2}$ ). 
Alguns periódicos têm conquistado a inclusão em bases de dados reputadas, como SciELO, Scopus e Web of Science (WoS). Isso implica em visibilidade dos artigos publicados em processos avaliativos, o que leva à atração de mais submissões ao periódico. A reputação conquistada, contudo, não é perene, nem garante melhor qualidade editorial ampla.

Outras qualidades importam, além da visibilidade. Blattmann e Santos (2014) abordam a continuidade e pontualidade de periódicos, citando a descontinuidade da revista ex-líder, homônima da área, Ciência da Informação, que recentemente passou vários anos interrompida.

Carelli e Giannasi-Kaimen (2009) julgam satisfatório o desempenho de revistas brasileiras líderes nesta área em aspectos como equipe editorial, presença em mecanismos de indexação, instruções para autores, usabilidade do sítio web etc. Isso, no entanto, contrasta com nossa experiência recente, pois encontramos moratória de submissões e discrepâncias em instruções aos autores nessas revistas, até no estrato Qualis A1.

Interromper submissões é uma intervenção para tentar evitar o hipercrescimento e colapso (overshoot and collapse), efeito conhecido na dinâmica de sistemas (STERMAN, 2000). Nesse padrão de comportamento, um sistema cresce de modo insustentável até a ruptura.

Nas revistas brasileiras líderes da área da Ciência da Informação, o colapso é previsível conforme o modelo de negócio vigente: crescimento do sistema de pós-graduação e pressão produtivista que atraem mais e mais submissões sem adequar os recursos à disposição dessas revistas.

Para explorar esse contexto, o presente estudo apresenta uma proposta de um modelo de dinâmica de sistemas que representa estoques e fluxos de artigos em etapas do processo editorial. Nessa proposta, são conjeturados 22 cenários alternativos, com oferta distinta de submissões, com ou sem intervenções (moratória de submissões e aumento agudo de rejeições preliminares).

Este artigo amplia a comunicação oral (KERN; URIONA, 2018). apresentada no $6^{\circ}$ Encontro Brasileiro de Bibliometria e Cientometria $\left(6^{\circ}\right.$ EBBC). Aqui, aprofundam-se a fundamentação e os procedimentos, a coleta de dados é detalhada e a simulação de cenários é refeita, com 22 variações da 
sobrecarga das revistas brasileiras líderes em Ciência da Informação. As próximas seções apresentam fundamentos da dinâmica de sistemas, os aspectos metodológicos, os resultados e as considerações finais.

\section{Simulação com dinâmica de sistemas}

A dinâmica de sistemas, criada por Jay W. Forrester, do Massachusetts Institute of Technology (MIT), na década de 1950, é uma abordagem para estudar o comportamento de um sistema no tempo por meio de simulação em ambiente computacional (SYSTEM DYNAMICS SOCIETY, 2017).

Os construtos essenciais da dinâmica de sistemas são laços de causa e efeito, estoques e fluxos. O conceito de estoque é maleável - qualquer coisa que se conceba acumulável.

A técnica ganhou projeção com The Limits to Growth (MEADOWS et al., 1972), um estudo de cenários com macrovariáveis (ou estoques) como população, poluição e recursos não renováveis. A Figura 1 mostra 0 comportamento de algumas macrovariáveis no tempo: as linhas pontilhadas são projeções do estudo e as linhas grossas contínuas representam dados históricos. O modelo de simulação foi calibrado com dados até cerca de 1970, para o estudo original. Cerca de 30 anos depois, o estudo foi atualizado (MEADOWS; RANDERS; MEADOWS, 2004), revelando boa aproximação entre as projeções e os dados históricos nesse período.

Figura 1 - Simulação do comportamento de macrovariáveis em The Limits to Growth

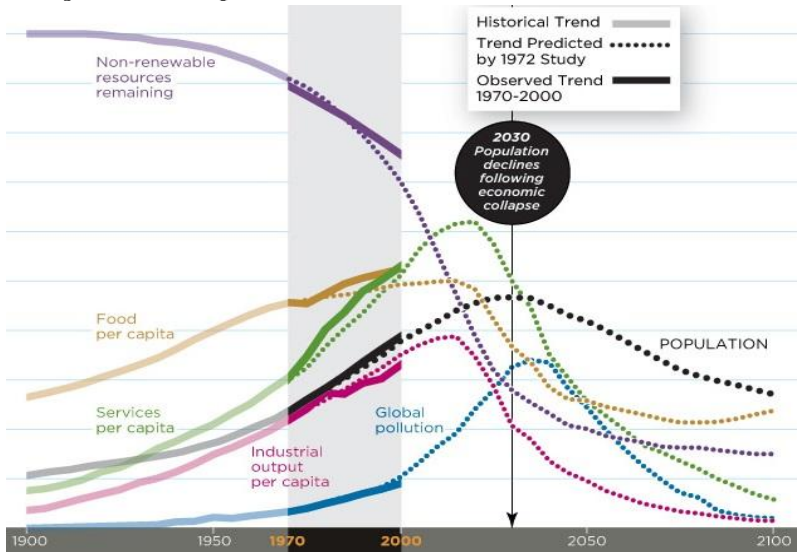

Fonte: Disponível em: <http://adrastia.org/manifest/> Acesso em: 23 set. 2018. 
O estudo ganhou seguidores e também críticos (por exemplo, Cole et al., 1973), mas esses não foram capazes de refutar os achados. The Limits to Growth foi um marco na compreensão de que nenhum sistema econômico pode crescer indefinidamente (MEADOWS; MEADOWS, 2007).

A simulação em dinâmica de sistemas permite gerar novos insights sobre o sistema, entender seu comportamento no tempo e propor ações de melhoria (STERMAN, 2000). Considerando que a proposta do presente estudo é elaborar um modelo de dinâmica de sistemas, a pesquisa demandou levantar dados históricos para conhecer o comportamento anterior do fluxo de artigos em revistas brasileiras de Ciência da Informação e, então, construir cenários do comportamento futuro. $\mathrm{O}$ detalhamento dos materiais e métodos desta pesquisa é apresentado a seguir.

\section{Procedimentos metodológicos}

Os materiais da pesquisa são dados históricos de processos editoriais e um software de modelagem e simulação em dinâmica de sistemas. O software usado foi Stella Architect 1.5 (<www.iseesystems.com>).

Os dados empíricos para estimar quantidades de estoques e fluxos do processo editorial vêm da seção Estatísticas nos sítios das revistas. Foram coletados dados sobre número de submissões, artigos enviados para revisão por pares, aceites, rejeições e tempos até aceite e publicação para todas as revistas brasileiras primárias ativas de Ciência da Informação nos estratos Qualis (20132016) A1, A2 e B1 da Capes (<qualis.capes.gov.br>).

Os dados para estimar parâmetros dos estoques e fluxos no sistema editorial foram obtidos por um levantamento nos sítios das revistas entre 2 e 13 de dezembro 2017. Já a elaboração de um modelo de dinâmica de sistemas é atividade artesanal e iterativa. É uma modelagem ou abstração informada por dados empíricos, embora possa utilizar estimativas. De acordo com Randers (1980), os modelos de dinâmica de sistemas são criados em quatro estágios: conceituação, formulação, testes e implementação. 
Conceituar implica definir um propósito para o modelo, seus limites e variáveis-chave e o comportamento dessas variáveis. Também inclui criar um diagrama usando os construtos básicos - laços de causa e efeito, estoques e fluxos (a seção Resultados, à frente, mostra diagramas de dinâmica de sistemas).

Formular é associar equações matemáticas a elementos do modelo, bem como estimar e selecionar os valores dos parâmetros. Testar é iniciar a simulação, verificando o comportamento das variáveis no tempo e sua sensibilidade a perturbações nas equações e parâmetros.

Implementar é consolidar equações e valores de parâmetros e registrar a resposta do modelo a diferentes políticas ou cenários. A partir destes procedimentos, a próxima seção apresenta o modelo construído e 22 cenários de funcionamento de um periódico brasileiro de Ciência da Informação (hipotético, não específico) sob parâmetros diversos de oferta de artigos submetidos e diferentes reações frente ao aumento do número de submissões.

\section{Resultados}

Apresenta-se a seguir o levantamento empírico empreendido para arbitrar quantidades de estoques e fluxos do processo editorial, a modelagem em dinâmica de sistemas e as simulações de cenários da sobrecarga das revistas e suas reações editoriais para aliviar essa sobrecarga.

\subsection{Dados empíricos para estimar estoques e fluxos do processo editorial}

Identificamos três revistas brasileiras primárias ativas de Ciência da Informação no estrato Qualis A1 ${ }^{3}$ (área Comunicação e Informação, quadriênio 2013-2016), outras três revistas em $\mathrm{A} 2^{4}$ e 12 em B1 ${ }^{5}$. Algumas exibem estatísticas, e.g., de submissões, envios para revisão, aceites, rejeições, tempo de avaliação e tempo até publicação, conforme a figura 2. 
Figura 2 - Disponibilidade de estatísticas nos sítios das revistas sobre artigos processados

\begin{tabular}{|c|c|c|c|c|c|c|c|c|c|c|c|c|c|}
\hline Revista & Qualis & 2006 & 2007 & 2008 & 2009 & 2010 & 2011 & 2012 & 2013 & 2014 & 2015 & 2016 & $2017^{*}$ \\
\hline Inf \& Soc & \begin{tabular}{|l|}
$\mathrm{A} 1$ \\
\end{tabular} & & & & & & & & & & & & \\
\hline Persp Cl & A1 & & SPARTP & SPARtP & SPARTP & SPAR AA TP & SPARTA TP & SPAR tA IP & SPARtP & SPAR tA IP & SPARtA tP & SPAR tA IP & SPARtP \\
\hline Transinf & A1 & & & & & & & & & & & & \\
\hline Em Quest & $A 2$ & & & & & & & & & & & & \\
\hline Enc Bib & A2 & & SPARtP & SPARtP & SPARtP & SPARTA & SPARTP & SPARTA & SPARtP & SPARTP & SPAR tA tP & SPAR tA tP & SPAR AA TP \\
\hline $\operatorname{lnf} \& \ln f$ & A2 & $\mathrm{s}$ & $\mathrm{s}$ & \begin{tabular}{|l|}
$s$ \\
\end{tabular} & s & $s$ & $s$ & s & s & $\mathrm{s}$ & $\mathrm{s}$ & $\mathrm{s}$ & $s$ \\
\hline Agora & B1 & & & & & & SPARtP & SPARtP & SPARtP & SPARTP & SPARTA IP & SPARTA TP & SPAR TA TP \\
\hline BJIS & B1 & & & & & & & & & & & & \\
\hline Cilnf & B1 & & & & & & & & & & & & \\
\hline Incid & B1 & & & & & & & & & & & & \\
\hline Liinc & B1 & s & SPAR & SPAR & SPAR & SPAR & SPAR & SPAR & SPAR & SPAR & SPAR & SPAR & SPAR \\
\hline Museol Patr & B1 & & & & & & & & & & & & \\
\hline Persp G\&C & B1 & & & & & & & & & & & & \\
\hline PdAcesso & B1 & & s & s & s & s & $\mathrm{s}$ & s & $s$ & s & $\mathrm{s}$ & $s$ & $s$ \\
\hline Rbbd & B1 & & & & & & & & & & & & \\
\hline Rdbci & B1 & & & & & & & & & & SPARtP & SPAR RA IP & SPAR TA TP \\
\hline Recils & B1 & & & & & & & & & & SPAR IA IP & SPAR RA IP & S PA R tA tP \\
\hline Rici & B1 & & & & & SPARtP & SPARtP & SPARtP & SPAR tA TP & $S P A R$ tA tP & SPARtP & SPAR RA tP & SPARtA IP \\
\hline \multirow[t]{2}{*}{ Notaçãa: } & s & subr & sôes & & & aceites & tA & tempo de & valiação & & & Coleta em 2- & $-13 / 12 / 2017$ \\
\hline & P & artigos & srevisados & sporpares & $\mathrm{R}$ & rejeições & tP & tempo até $p$ & publicação & & & & \\
\hline
\end{tabular}

Fonte: Dados da pesquisa (2018).

As quantidades anuais de submissões estão na Figura 3. Foram calculados valores médios mensais de submissões para cada ano em cada revista. Dentre os valores disponíveis, encontram-se medianas de médias mensais de submissões de 16,3 $(\mathrm{n}=1)$ para revistas A1, 7,9 $(\mathrm{n}=2)$ para A2 e 3,8 $(\mathrm{n}=6)$ para B1.

Figura 3 - Número de submissões anuais por revista

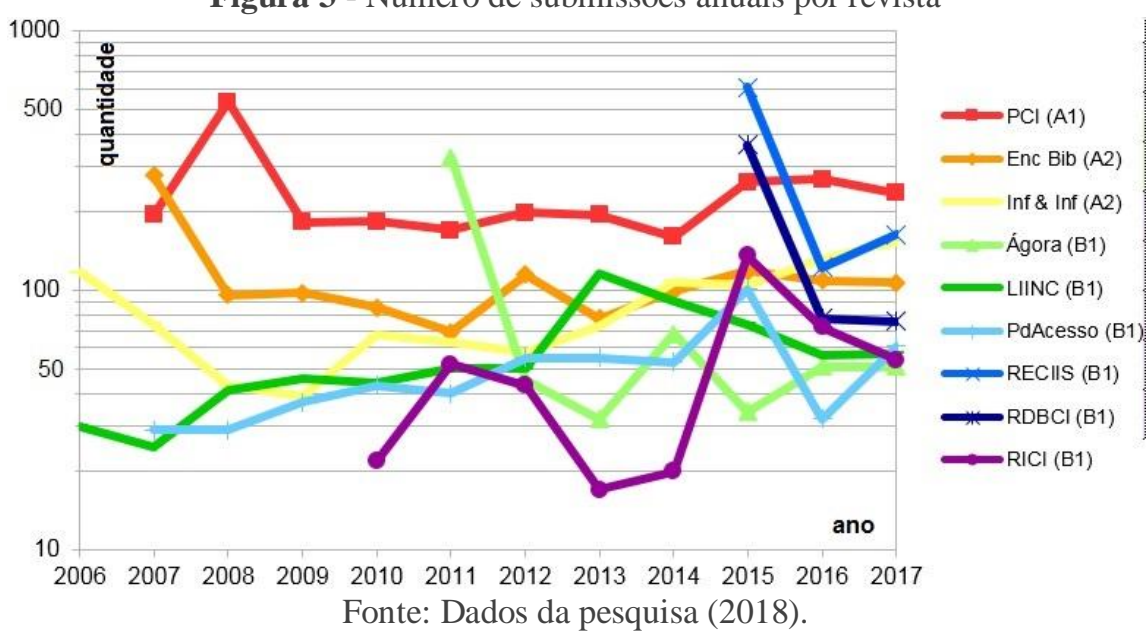

Foi adotado o valor de 16,3, mediana das médias mensais de submissões à única revista Qualis A1 (Perspectivas em Ciência da Informação) que as reporta, como referência para as simulações apresentadas adiante. Corresponde a uma quantidade anual de 196 submissões, ocorridas em 2007. Ao adotar esse 
valor, não se pretende representar uma revista específica, mas estimar um valor típico para revistas brasileiras líderes na área.

Alguns artigos submetidos são rejeitados pelos editores na triagem preliminar, enquanto outros são submetidos à revisão por pares. Foram calculados valores medianos para as médias mensais de artigos enviados para revisão, de 7,8 $(n=1)$ para revistas $A 1,4,8(n=1)$ para $A 2$ e 2,8 $(n=5)$ para B1. Os números anuais estão na figura 4.

Figura 4 - Quantidade anual de artigos avaliados por pares por revista

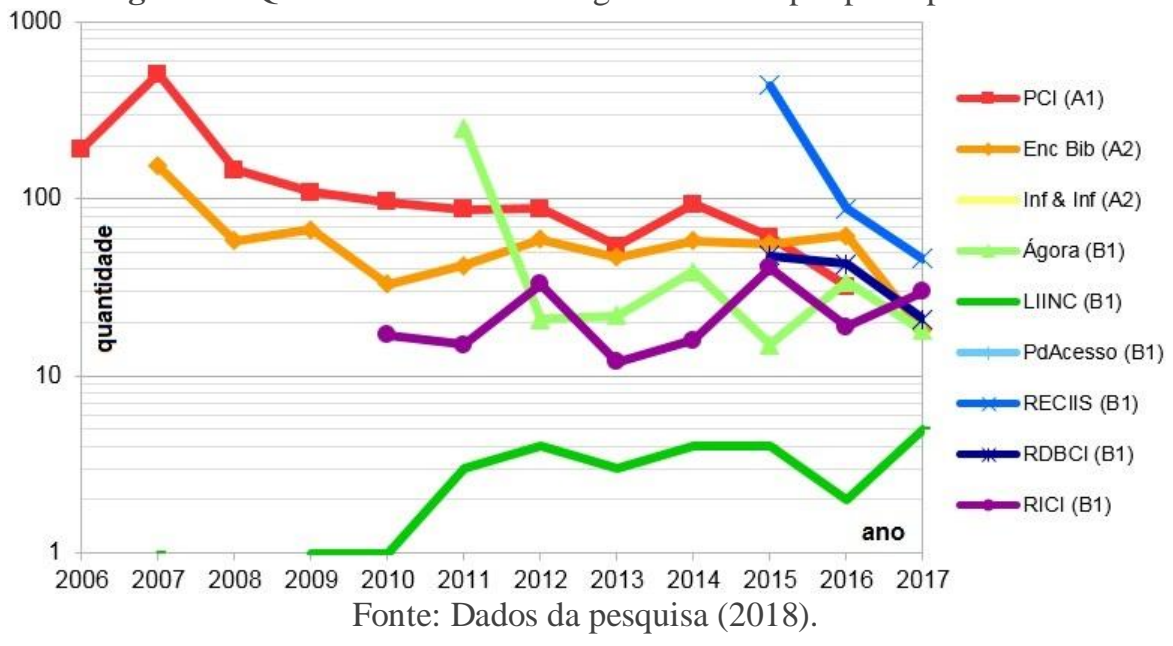

O número de rejeições de artigos na triagem por editores não faz parte das estatísticas publicadas pelas revistas. No entanto, parece plausível adotar a diferença entre o número de submissões e o de envios para revisão por pares como medida da quantidade dessas rejeições preliminares. A Figura 5 mostra esses valores anuais por revista.

Figura 5 - Quantidade anual por revista de submissões menos avaliações por pares

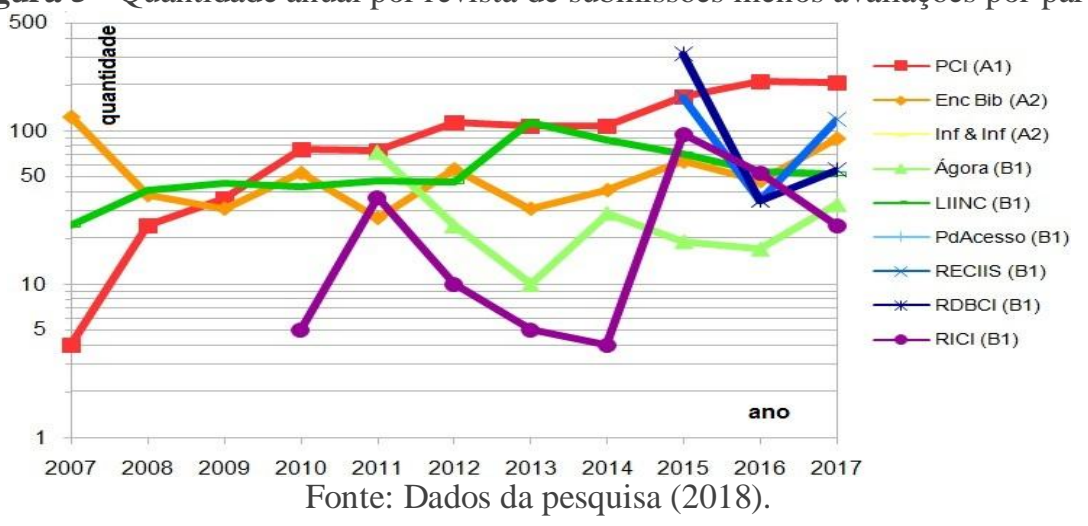


O tempo total entre a submissão e a decisão editorial definitiva é uma medida relevante da qualidade do processo editorial (ALPERIN, 2018), já que a novidade é colocada em risco quando artigos são retidos por longos períodos de tempo até uma decisão pela publicação ou rejeição. Silva e Dobránszki (2017) consideram adequado um tempo de cinco a oito meses até a decisão final. A Figura 6 mostra esses tempos em dias para cada revista.

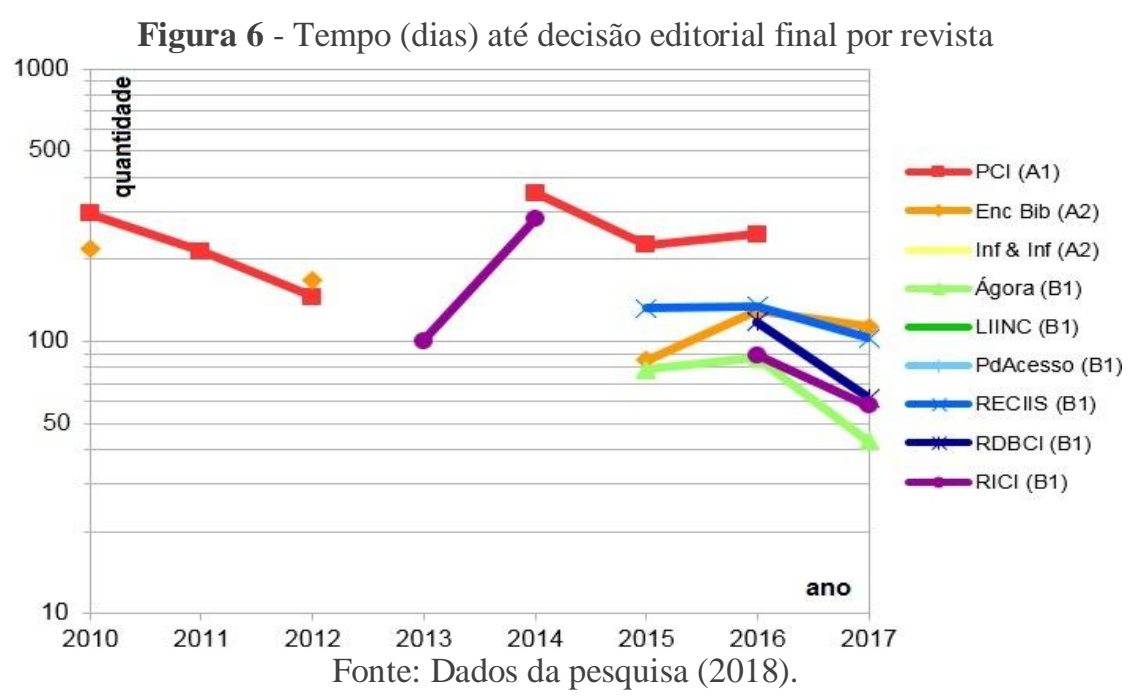

O levantamento recém-apresentado descreve a realidade dos processos editoriais das revistas brasileiras líderes na Ciência da Informação. Essa realidade inspira a definição de parâmetros para estoques e fluxos do processo editorial, conforme a modelagem a seguir.

\subsection{Modelo de dinâmica de sistemas}

O modelo em dinâmica de sistemas foi elaborado com o propósito de representar e simular o comportamento de estoques e fluxos do processo editorial entre a submissão de artigos e a decisão editorial final pela rejeição ou publicação. Na Figura 7, os estoques são representados em caixas que mostram os artigos aguardando na fila de triagem, ou Desk Review (com estoque inicial de 36 e tempo previsto de três meses) e Artigos em Avaliação (com estoque inicial de 36 e tempo previsto de quatro meses). 
Figura 7 - Modelo de simulação do processo de avaliação de artigos

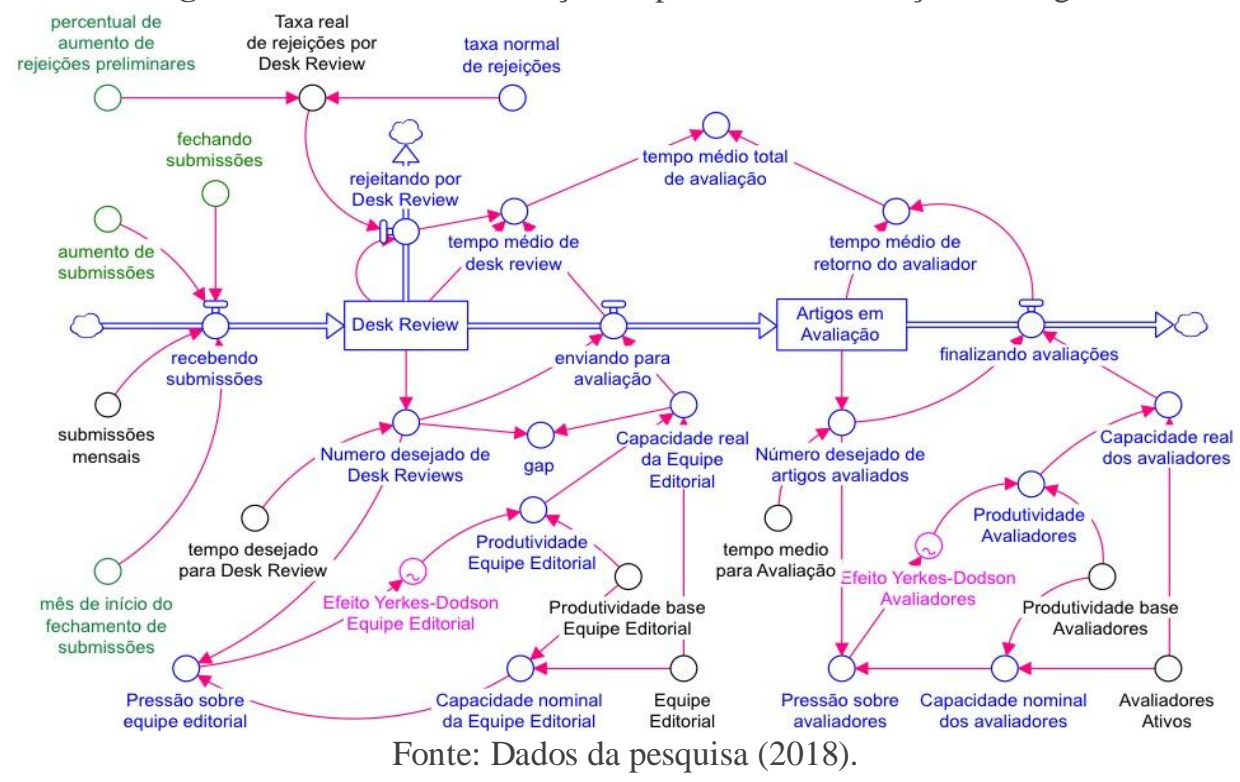

Os fluxos, representados graficamente como registros em azul, podem ser lidos da seguinte forma: no ramo principal do modelo, na horizontal, os novos artigos chegam na triagem de submissões (Desk Review) em taxa inicial média de 16,3 submissões por mês. Alguns são rejeitados (taxa inicial de 20\%) e outros entram em revisão por pares (Artigos em Avaliação), até haver uma decisão editorial final. O fluxo é regulado pelos valores dos parâmetros, com valores iniciais na Tabela 1, arbitrados a partir dos dados empíricos anteriores, obtidos nas estatísticas das revistas, a serem alterados nas simulações mostradas adiante.

Tabela 1 - Parâmetros (valores iniciais) arbitrados para as simulações de cenários

\section{Parâmetro}

Aumento de submissões

Avaliadores ativos

Equipe editorial

Fechando submissões

Artigos em avaliação

Desk review

Mês de início do fechamento de submissões

$\%$ aumento de rejeições preliminares

Produtividade base - Avaliadores

Produtividade base - Equipe editorial

Submissões mensais

Taxa normal de rejeições

Tempo desejado para desk review

Tempo médio para avaliação

\section{Valor Unidade}

144 avaliador

2 editor

0 artigo/mês

36 artigo

36 artigo

24 mês

$0 \%$

0,1 art./avaliador/mês

4 artigos/editor/mês

16,3 artigos/mês

$20 \%$

3 mês

4 mês

Fonte: Dados da pesquisa (2018). 
Além dos estoques e fluxos, os círculos na Figura 7 representam parâmetros (em preto), variáveis auxiliares (em azul), funções de transformação não-lineares (em rosa) e variáveis opcionais para representar alterações no fluxo editorial (em verde). As setas em magenta são dependências entre variáveis e outras variáveis e parâmetros. As nuvens estilizadas são fronteiras do modelo entradas ou saídas fora de seu escopo.

As funções de transformação não lineares (círculos em rosa na Figura 7) regulam a flexibilidade da produtividade de editores e revisores. $\mathrm{O}$ aumento de submissões pressiona a capacidade de equipe editorial e avaliadores, que aumentam sua produtividade até certo limite, o que é uma propriedade conhecida de sistemas de serviços (OLIVA; STERMAN, 2001; LÓPEZ; ZÚÑIGA, 2014). Porém, há um ponto a partir do qual a produtividade pressionada começa a cair e prenuncia o colapso, o que constitui o efeito Yerkes-Dodson (RUDOLPH; REPENNING, 2002).

Esse efeito foi modelado na

Figura 8. A pressão sobre os avaliadores (eixo horizontal) é a razão entre o número desejado de revisões e a capacidade nominal do avaliador. Logo, se a demanda corresponde à capacidade nominal de 0,1 revisões por mês conforme a Tabela 1, a pressão é 1. À medida que a pressão cresce, revisores e avaliadores respondem aumentando sua capacidade de trabalho em até 200\% (revisores) ou $250 \%$ (editores), mas há um limite além do qual o desempenho começa a degradar-se, chegando à fadiga, com queda de produção, ou mesmo ao esgotamento (burnout).

Figura 8 - Efeito da pressão sobre a produtividade de (A) avaliadores e (B) equipe editorial de acordo com o efeito Yerkes-Dodson
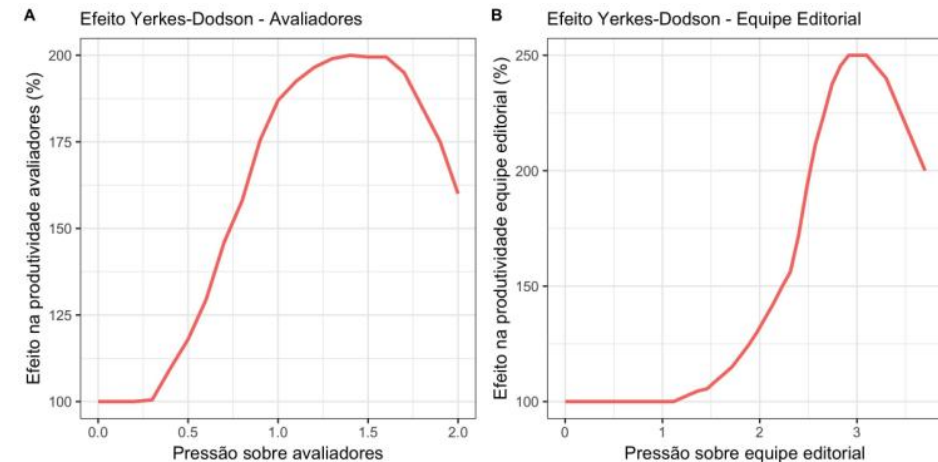

Fonte: Dados da pesquisa (2018). 
Esses são parâmetros e equações que regulam os estoques e fluxos no modelo. A próxima seção apresenta vários cenários ou configurações distintas dos parâmetros para simular o comportamento do sistema.

\subsection{Estudo de cenários}

Criamos três tipos de cenários para estudar o comportamento do sistema. Todos têm como referência uma carga mensal de submissões de 16,3 artigos. Nos três cenários do tipo A, variam os níveis de aumento das submissões a partir do mês 12: da manutenção da carga mensal de 16,3 no cenário BAU (business-as-usual) a um aumento de submissões de $150 \%$ no cenário A3, conforme a Figura 9, que modifica os valores iniciais na Tabela 1 .

Figura 9 - Desenho dos experimentos para os 22 cenários estudados

\begin{tabular}{|c|c|c|c|c|c|}
\cline { 3 - 5 } \multicolumn{2}{c|}{} & $0 \%$ & $50 \%$ & $100 \%$ & $150 \%$ \\
\hline A & Aumento de submissões & BAU & A1 & A2 & A3 \\
\hline
\end{tabular}

\begin{tabular}{|c|c|c|c|c|c|}
\hline & & & \multicolumn{3}{|c|}{ Aumento de submissões } \\
\hline & & & $50 \%$ & $100 \%$ & $150 \%$ \\
\hline \multirow{3}{*}{$\mathrm{R}$} & \multirow{3}{*}{ Surto de Rejeições } & $10 \%$ & R1 & R4 & R7 \\
\hline & & $25 \%$ & $\mathrm{R} 2$ & R5 & R8 \\
\hline & & $50 \%$ & R3 & R6 & R9 \\
\hline
\end{tabular}

\begin{tabular}{|c|c|c|c|c|c|}
\hline & & & \multicolumn{3}{|c|}{ Aumento de submissões } \\
\hline & & & $50 \%$ & $100 \%$ & $150 \%$ \\
\hline \multirow{3}{*}{ M } & \multirow{3}{*}{$\begin{array}{c}\text { Moratória de } \\
\text { submissões }\end{array}$} & 3 meses & M1 & M4 & M7 \\
\hline & & 6 meses & $\mathrm{M} 2$ & M5 & M8 \\
\hline & & 9 meses & M3 & M6 & M9 \\
\hline
\end{tabular}

Fonte: Dados da pesquisa (2018).

Nos nove cenários do tipo R, além do aumento de submissões, o sistema sofre uma intervenção editorial: o surto de rejeições na triagem, da ordem de 10 , 25 ou $50 \%$. Nos nove cenários do tipo M, a moratória (fechamento) de submissões durante 3, 6 ou 9 meses é combinada com diversos níveis de aumento das submissões. No total, são 22 cenários simulados por 60 meses (cinco anos), resultando nos valores finais de variáveis na Tabela 2. 
Tabela 2 - Resultados de variáveis selecionadas para os 22 cenários simulados ao fim dos 60 meses

\begin{tabular}{|c|c|c|c|c|c|c|c|}
\hline $\begin{array}{l}\text { Ce- } \\
\text { ná- } \\
\text { rio }\end{array}$ & $\begin{array}{l}\text { Submis- } \\
\text { sões por } \\
\text { mês }\end{array}$ & $\begin{array}{l}\text { Artigos envia- } \\
\text { dos para re- } \\
\text { visão por mês }\end{array}$ & $\begin{array}{l}\text { Rejeições } \\
\text { preliminares } \\
\text { por mês }\end{array}$ & $\begin{array}{l}\text { Artigos } \\
\text { revisados } \\
\text { por mês }\end{array}$ & $\begin{array}{c}\text { Artigos } \\
\text { aguardando } \\
\text { desk review }\end{array}$ & $\begin{array}{l}\text { Artigos em } \\
\text { avaliação }\end{array}$ & $\begin{array}{c}\text { Tempo total } \\
\text { de avaliação } \\
\text { (meses) }\end{array}$ \\
\hline BAU & 16,30 & 8,77 & 7,53 & 8,77 & 37,65 & 35,08 & 6,30 \\
\hline $\mathrm{A} 1$ & 24,45 & 13,08 & 11,37 & 13,08 & 56,84 & 54,93 & 6,52 \\
\hline $\mathrm{A} 2$ & 32,60 & 19,26 & 13,34 & 14,40 & 66,70 & 268,20 & 20,65 \\
\hline A3 & 40,75 & 20,00 & 20,75 & 14,40 & 103,75 & 307,00 & 23,85 \\
\hline $\mathrm{R} 1$ & 24,45 & 10,34 & 14,11 & 10,34 & 47,03 & 41,36 & 5,92 \\
\hline $\mathrm{R} 2$ & 24,45 & 8,50 & 15,95 & 8,50 & 35,45 & 34,00 & 5,44 \\
\hline R3 & 24,45 & 7,89 & 16,56 & 7,89 & 23,66 & 31,55 & 4,96 \\
\hline $\mathrm{R} 4$ & 32,60 & 15,00 & 17,59 & 14,36 & 59,20 & 88,21 & 7,94 \\
\hline R5 & 32,60 & 10,62 & 21,99 & 10,78 & 48,86 & 43,11 & 5,49 \\
\hline R6 & 32,60 & 8,43 & 24,17 & 8,43 & 34,76 & 33,73 & 5,06 \\
\hline $\mathrm{R} 7$ & 40,75 & 19,91 & 20,84 & 14,40 & 69,46 & 299,38 & 22,48 \\
\hline $\mathrm{R} 8$ & 40,75 & 14,39 & 26,36 & 14,23 & 58,57 & 71,76 & 6,48 \\
\hline $\mathrm{R} 9$ & 40,75 & 9,77 & 30,98 & 9,77 & 44,25 & 39,09 & 5,08 \\
\hline M1 & 24,45 & 13,08 & 11,37 & 13,03 & 56,84 & 54,60 & 6,51 \\
\hline M2 & 24,45 & 13,08 & 11,37 & 13,02 & 56,84 & 54,51 & 6,51 \\
\hline M3 & 24,45 & 13,08 & 11,37 & 13,02 & 56,84 & 54,48 & 6,51 \\
\hline M4 & 32,60 & 19,26 & 13,34 & 14,40 & 66,70 & 206,36 & 16,37 \\
\hline M5 & 32,60 & 19,26 & 13,34 & 14,40 & 66,70 & 174,42 & 14,15 \\
\hline M6 & 32,60 & 19,26 & 13,34 & 14,40 & 66,70 & 169,27 & 13,79 \\
\hline M7 & 40,75 & 20,00 & 20,75 & 14,40 & 103,63 & 270,75 & 21,33 \\
\hline M8 & 40,75 & 20,00 & 20,75 & 14,40 & 103,61 & 215,35 & 17,49 \\
\hline M9 & 40,75 & 20,00 & 20,75 & 14,40 & 103,61 & 196,56 & 16,18 \\
\hline
\end{tabular}

Fonte: Dados da pesquisa (2018).

Os valores em vários cenários indicam uma difícil sustentabilidade da revista. No modelo de negócio usual nas revistas estudadas, é difícil aumentar a equipe editorial ou o corpo de revisores. Assim, parecem inadequados o cento de artigos em espera por desk review (cenários M7-M8-M9), as várias centenas de artigos em avaliação (A2-A3-R7-M4-M7-M8) e os tempos de espera por uma decisão editorial superiores a um ano (nove dos 22 cenários).

Convém observar o comportamento do sistema no tempo e não apenas o resultado após 60 meses. Nos quatro cenários sem intervenções editoriais abruptas, na Figura 10, observa-se uma estabilidade no cenário BAU, que recebe uma carga mensal de 16,3 submissões. Mesmo o cenário A1, com mais 50\% de submissões a partir do mês 12, parece sustentável. Isso pode ser verdade para algumas revistas concretas, mas não certamente para todas. 
Figura 10 - Resultados da simulação nos cenários com aumento de submissões
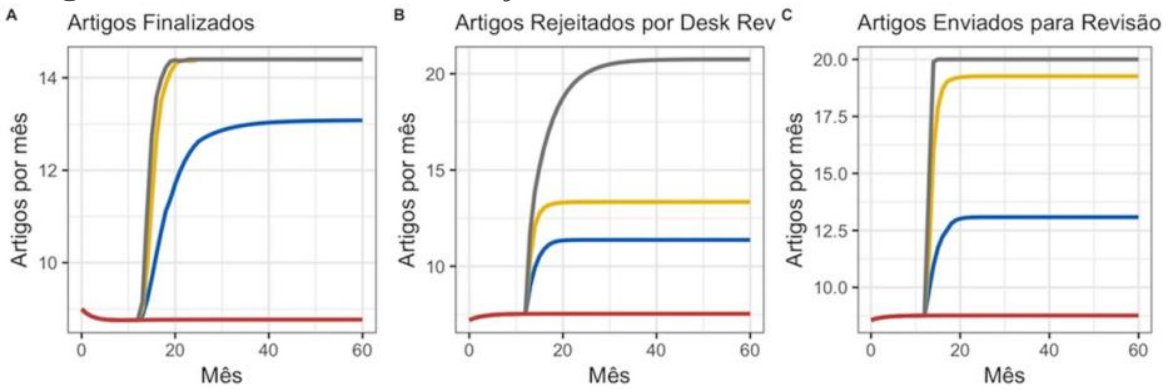

D Artigos aguardando Desk Reviev ${ }^{\mathrm{E}}$
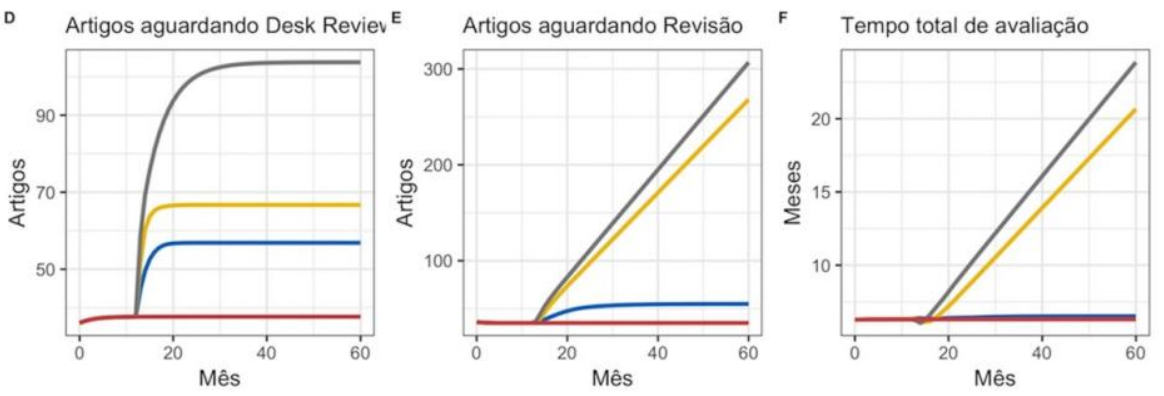

Cenário $-\mathrm{A}_{1}-\mathrm{A}_{2}-\mathrm{A}_{3}-\mathrm{BAU}$

Fonte: Dados da pesquisa (2018).

A figura 11 mostra o comportamento do sistema nos cenários do tipo $\mathrm{R}$, em que os aumentos na quantidade de submissões são enfrentados com aumento (surto) de rejeições na triagem. No cenário R4, a quantidade de artigos em espera por desk review chega a 70, ou 60 nos cenários R5 e R7. O tempo total de avaliação é claramente inviável para o cenário R4 e, embora esteja sob controle até o mês 60 , tende a tornar-se inviável no cenário R7.

Figura 11 - Resultados da simulação nos cenários com aumento de submissões e surto de

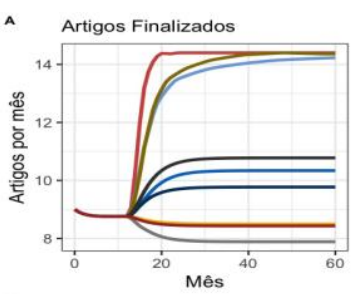
rejeições na triagem
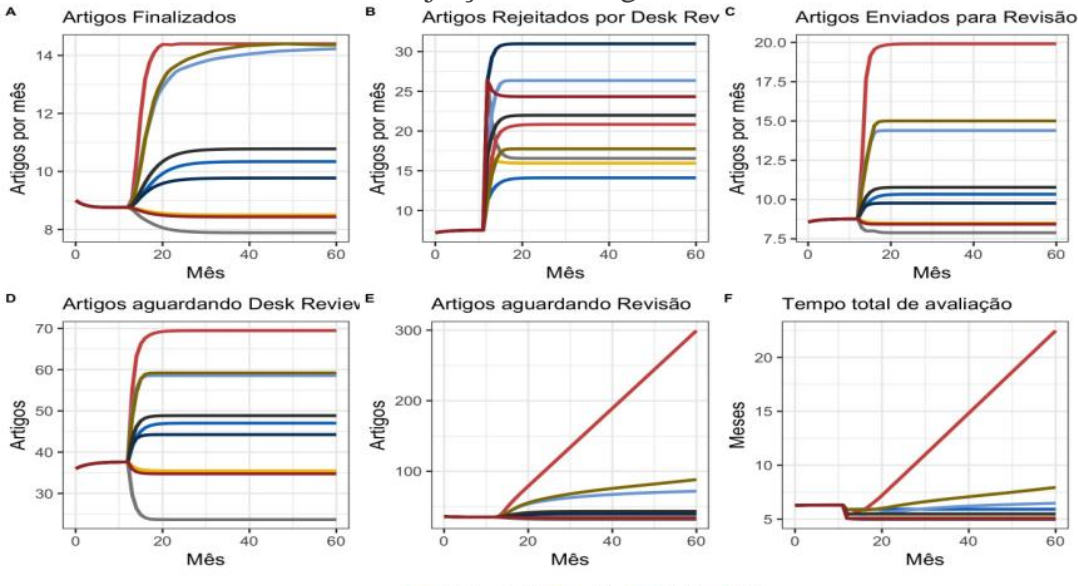

Cenário $-{ }_{\mathrm{R} 2}^{\mathrm{R} 1}$ - $_{\mathrm{R} 4}^{\mathrm{R}}$ Z $_{\mathrm{R} 6}^{\mathrm{R} 6}$ - $_{\mathrm{R} 8}^{\mathrm{R}}$

Fonte: Dados da pesquisa (2018). 
Finalmente, a figura 12 ilustra os cenários em que a revista responde às submissões crescentes impondo moratória de submissões a partir do mês 24 . A pressão sobre editores e revisores cresce a partir do aumento das submissões no mês 12. Essa pressão é aliviada no período de moratória, mas volta a crescer e o tempo de avaliação torna-se insustentável, exceto nos cenários M1, M2 e M3 (sobrepostos no gráfico), cuja carga mensal é de 24,45 submissões.

Figura 12 - Resultados da simulação nos cenários com aumento de submissões e moratória de submissões
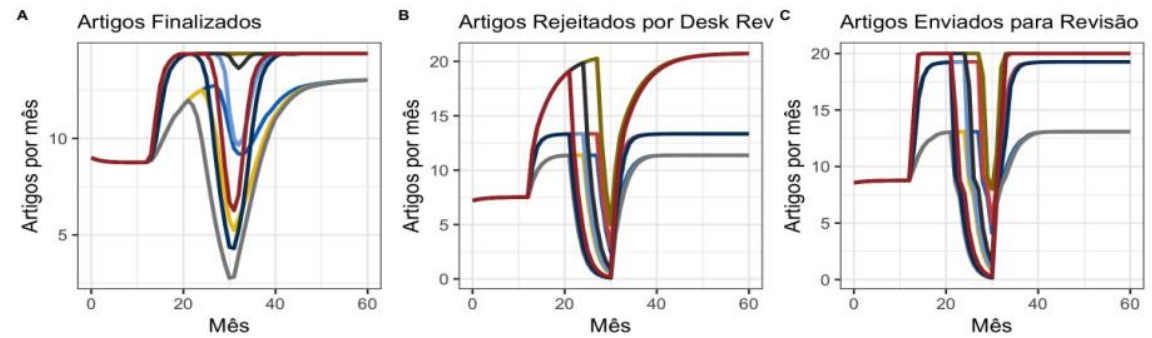

D Artigos aguardando Desk Revi€ $\epsilon^{\mathrm{E}}$
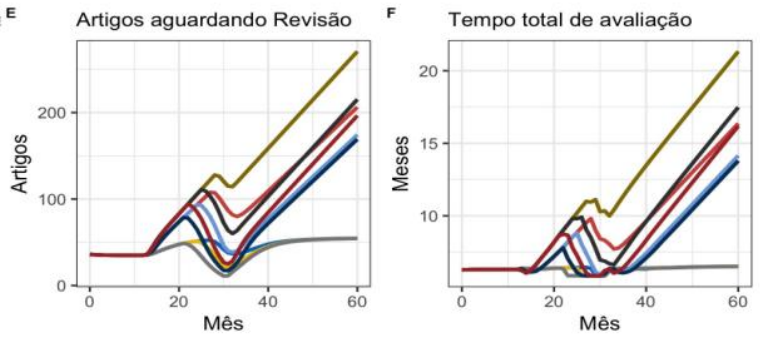

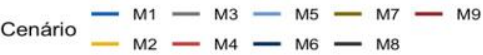

Fonte: Dados da pesquisa (2018).

\section{Considerações finais}

Neste artigo, exploramos o contexto da sobrecarga das revistas brasileiras líderes em Ciência da Informação por meio de simulações em dinâmica de sistemas, com risco variado de hipercrescimento e colapso. As simulações foram inspiradas em dados empíricos que, em alguns casos, surpreenderam os pesquisadores. Em particular, esperávamos o crescimento no número de submissões às revistas da área, dado o início recente de diversos cursos de mestrado e doutorado na área aliado ao recrudescimento da ênfase quantitativa nas avaliações em pós-graduação. Porém, não há um claro aumento do número de submissões às revistas, como se observa na Figura 3. 
Os 22 cenários estudados permitem simular o esgarçamento da capacidade de editores para tomar decisões de triagem e de revisores em dar pareceres sobre artigos submetidos. Os cenários espelharam três tipos de situações, todos com aumento de submissões: sem intervenções editoriais abruptas, com surto de rejeições na triagem e com moratória de submissões.

Uma demanda mensal de 16,3 novas submissões, plausível segundo os dados levantados nas estatísticas das revistas, foi tomada como ponto de partida. Alguns dos cenários estudados indicam a continuidade sustentável da revista, embora isso possa não ser válido para revistas específicas, já que tem sido frequente o fechamento de submissões por revistas com demanda bastante inferior às 16,3 submissões mensais.

Boa parte das simulações aponta para o hipercrescimento e colapso da revista. Em particular, intervenções editoriais como o surto de rejeições na triagem e a moratória de submissões aliviam momentaneamente a carga sobre editores e avaliadores, mas não garantem a sustentabilidade da revista no longo prazo.

Um dos riscos encontrados foi o tempo excessivo entre submissão e decisão editorial. Silva e Dobránszki (2017) propõem limites de tempo para cada estágio do processo de publicação, sem ultrapassar cinco a oito meses para uma decisão final. Também consideram que editores cometem negligência, senão má conduta, quando não comunicam ou não oferecem explicações apropriadas para atrasos.

O presente artigo se propõe a indicar tendências para revistas que seguem o modelo de negócio típico das revistas brasileiras de Ciência da Informação: em acesso aberto, sem taxas, mantido com recursos públicos, não escalável (isto é, o sucesso usualmente não implica em dotar a revista de melhores recursos). Não se propõe a fazer previsões precisas baseadas nos números apresentados, embora os relatos anedóticos que ouvimos sugira que os cenários são verossímeis.

Recentemente, durante a apresentação desta pesquisa (KERN; URIONA, 2018) no $6^{\circ} \mathrm{EBBC}$, um editor de revista afirmou seu enfrentamento constante do risco de colapso da revista que edita (de outra área do conhecimento); 
assumiu que trabalha com recursos muito escassos para uma revista mensal e bem reputada e que não pode sair em férias sob pena de não conseguir retomar o fluxo editorial normal após retornar. Se esta situação for comum, é plausível considerar que muitas revistas consomem um recurso não previsto e pouco renovável: a saúde dos editores.

O estudo é exploratório e tem limitações. As informações sobre o processo editorial são escassas. É incerta a confiabilidade das estatísticas de submissões e outras presentes nos sítios Web, já que poucas revistas as publicam - e de forma declarativa, não verificável.

São necessários mais estudos empíricos sobre a sobrecarga das revistas científicas brasileiras líderes na Ciência da Informação e suas estratégias cientificamente legítimas ou ilegítimas de enfrentamento. Desde já é possível tecer algumas conjeturas. As duas formas de enfrentamento do colapso sem dotação significativa de novos recursos estudadas neste artigo - moratória de submissões e aumento das rejeições na triagem - devem evoluir distintamente.

A moratória de submissões é anticientífica e muito visível. Logo, deve ser abolida, seja porque as revistas que a praticarem serão excluídas de bases de dados importantes, seja porque evitarão essa punição declarando nunca mais entrar em moratória. Já a rejeição na triagem (desk rejection) é diferente, pois pode ser legítima. O British Medical Journal, revista bem reputada e citada, aceita apenas 7\% das submissões e rejeita de 60 a $70 \%$ na triagem (GROVES; ABBASI, 2004). Um editor pode triar artigos por meio de uma avaliação rápida, porém rigorosa, ou pode rejeitar com base em subterfúgio (por exemplo, um injustificado "foge ao escopo da revista", ou uma rejeição na triagem após reter o artigo por muitos meses sem rejeitar nem encaminhar para revisão).

Distinguir formas legítimas e ilegítimas de enfrentar o aumento de submissões pode ser bastante difícil, ou impossível. A competência científica necessária para enfrentar os problemas editoriais é difícil de julgar, apesar da influência atual de medidas bibliométricas, já que impacto não é importância (CASADEVALL; FANG, 2015) e o próprio Garfield (2006, p. 92) preveniu quanto aos excessos do sucesso de indicadores métricos: "O uso de impactos de 
revistas na avaliação de indivíduos tem seus perigos inerentes. Num mundo ideal, os avaliadores leriam cada artigo e fariam julgamentos pessoais".

Em conclusão, os cenários estudados mostram uma tendência ao colapso ou à fadiga, colocando em risco o status conquistado pelas revistas. A cultura de avaliação requer indexação em bases de dados seletivas (NASCIMENTO; MUGNAINI, 2016), o que leva a revista aos estratos superiores do Qualis, mas isso pode privilegiar excessivamente algumas revistas (BARATA, 2016) e inflar exageradamente sua oferta de novas submissões. Sem dotação adequada de recursos para responder à demanda por avaliação competente nem formação adequada do corpo editorial (STUMPF, 1998), as revistas brasileiras líderes na Ciência da Informação ficam excessivamente dependentes de uns poucos editores sobrecarregados.

\section{Financiamento}

$\mathrm{O}$ primeiro autor (VMK) é bolsista de produtividade em pesquisa do $\mathrm{CNPq}$, nível 2, área Comunicação e Informação.

\section{Referências}

ALPERIN, Juan Pablo (@ juancommander). “I wouldn't think of it as a lesser journal. Any journal that takes 8 months to reply to an initial submission is not a good journal". 9:42 PM, 21 mar. 2018. Twitter. Disponível em:

https://twitter.com/juancommander/status/976620158314663936. Acesso em: 18 dez. 2018.

BARATA, Rita de Cássia Barradas. Dez coisas que você deveria saber sobre o Qualis. Revista Brasileira de Pós-Graduação, v. 13, n. 30, p. 13-40, 2016.

BLATTMANN, Ursula; SANTOS, Raimundo Nonato Macedo dos. Revistas científicas brasileiras e sua visibilidade no acesso aberto. Informação \& Sociedade, v. 24, n. 3, p. 99-106, 2014.

CARELLI, Ana Esmeralda; GIANNASI-KAIMEN, Maria Júlia. Os periódicos científicos no compartilhamento da informação e do conhecimento: aspectos extrínsecos dos periódicos eletrônicos Qualis A da área de ciência da informação. Encontros Bibli, v. 14, n. 27, 2009. 
CASADEVALL, Arturo; FANG, Ferric C. Impacted science: impact is not importance. mBio, v. 6, n. 5, 2015.

COLE, Hugh S. D.; FREEMAN, C.; JAHODA, M.; PAVITT, K. L. Thinking about the future: a critique of The Limits to Growth. Londres: Chatto \& Windus, 1973.

FERREIRA, Aurélio Buarque de Hollanda. Novo Aurélio Século XXI: o dicionário da língua portuguesa. 3. ed. rev. ampl. Rio de Janeiro: Nova Fronteira, 1999.

GARFIELD, Eugene. The history and meaning of the journal impact factor. Journal of the American Medical Association, v. 295, n. 1, p. 90-93, 2006.

GROVES, Trish; ABBASI, Kamran. Screening research papers by reading abstracts: Please get the abstract right, because we may use it alone to assess your paper. BMJ: British Medical Journal, v. 329, n. 7464, p. 470-471, 2004.

KERN, Vinícius Medina; URIONA MALDONADO, Mauricio.

Hipercrescimento e colapso: Conjeturando cenários da sobrecarga das revistas científicas brasileiras líderes na Ciência da Informação. In: ENCONTRO BRASILEIRO DE BIBLIOMETRIA E CIENTOMETRIA (EBBC), 6, 2018, Rio de Janeiro. Anais... Rio de Janeiro,RJ: UFRJ, 2018. p. 90-98.

LÓPEZ, Luis; ZÚÑIGA, Roy. Dynamics of judicial service supply chains. Journal of Business Research, v. 67, n. 7, p. 1447-1454, 2014.

MEADOWS, Donella H.; MEADOWS, Dennis L. The history and conclusions of The Limits to Growth. System Dynamics Review, v. 23, n. 2-3, p. 191-197, 2007.

MEADOWS, Donella H.; MEADOWS, Dennis L.; RANDERS, Jørgen; BEHRENS III, William W. The limits to growth: a report for The Club of Rome's project on the predicament of mankind. New York: Universe Books, 1972. Disponível em: http://www.donellameadows.org/wpcontent/userfiles/Limits-to-Growth-digital-scan-version.pdf. Acesso em: 23 set. 2018.

MEADOWS, Donella H.; RANDERS, Jørgen; MEADOWS, Dennis L. The limits to growth: the 30-year update. White River Junction-VT: Chelsea Green Publishing, 2004.

NASCIMENTO, Carla Cristina do; MUGNAINI, Rogerio. Qualidade de revistas científicas: um estudo da literatura publicada entre 2003 e 2013. In: ENCONTRO NACIONAL DE PESQUISA EM CIÊNCIA DA INFORMAÇÃO (ENANCIB), 17., 2016, Salvador. Anais... Salvador-BA: UFBA; ANCIB, 2016.

OLIVA, Rogelio; STERMAN, John D. Cutting corners and working overtime: quality erosion in the service industry. Management Science, v. 47, n. 7, p. 894-914, 2001. 
RANDERS, Jørgen. Elements of the system dynamics method. Portland-OR: Productivity Press, 1980.

RUDOLPH, Jenny W.; REPENNING, Nelson P. Disaster dynamics: understanding the role of quantity in organizational collapse. Administrative Science Quarterly, v. 47, p. 1-30, 2002.

SILVA, Jaime A. Teixeira da; DOBRÁNSZKI, Judit. Excessively long editorial decisions and excessively long publication times by journals: causes, risks, consequences, and proposed solutions. Publishing Research Quarterly, v. 33, n. 1, p. 101-108, 2017.

STERMAN, John D. Business dynamics: systems thinking and modeling for a complex world. Boston: McGraw-Hill, 2000.

SYSTEM DYNAMICS SOCIETY. What is SD: Introduction to system dynamics. Albany, NY, EUA: System Dynamics Society, 2018. Disponível em https://www.systemdynamics.org/what-is-sd. Acesso em: 20 set. 2018.

STUMPF, Ida Regina Chittó. Reflexões sobre as revistas brasileiras. Intexto, v. 1, n. 3, p. 1-10, 1998.

\title{
Scenarios of "overshoot \& collapse" dynamics in leading Brazilian journals in Information Science
}

\begin{abstract}
Brazilian journals in Information Science typically depend on semivoluntary work, with scarce resources. The inclusion of some of these in reputable databases gives them status and attracts more and more submissions, without resource scalability. This article explores this context through a system dynamics model that represents stocks and flows of papers throughout the editorial process. We run 22 alternative scenarios, with different submissions, with or without interventions (interruption of submissions and sharp increase in desk rejections). Several of these scenarios indicate the journal's imminent risk of collapsing, regardless of interventions that alleviate the burden only momentarily. Few overburdened editors sustain the system's continuity.
\end{abstract}

Keywords: Journalology. Qualis Capes. Academic productivism. Peer review. Scholarly publishing.

Recebido: $25 / 09 / 2018$ Aceito: $03 / 12 / 2018$ 


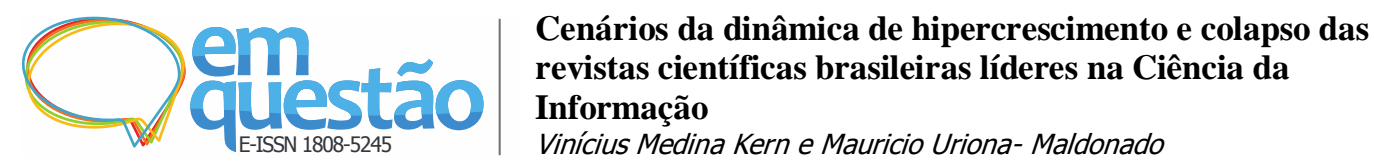

\footnotetext{
${ }^{1}$ Disponível em: <https://pkp.sfu.ca/ojs/>. Acesso em: 17 dez. 2018.

${ }^{2}$ Disponível em: <http://www.ibict.br/pesquisa-desenvolvimento-tecnologico-e-inovacao/sistemaeletronico-de-editoracao-de-revistas-seer>. Acesso em: 17 dez. 2018.

${ }^{3}$ Revistas Qualis A1 na Figura 2: Informação \& Sociedade (Inf. \& Soc.), Perspectivas em Ciência da Informação (Persp. CI) e Transinformação (Transinf).

${ }^{4}$ Revistas Qualis A2 na Figura 2: Em Questão (Em Quest), Encontros Bibli (Enc. Bib.) e Informação \& Informação (Inf \& Inf).

${ }^{5}$ Revistas Qualis B1 na Figura 2: Ágora, Brazilian Journal of Information Science (BJIS), Ciência da Informação (Ci. Inf), InCID: Revista de Ciência da Informação e Documentação (Incid), Liinc em Revista (Liinc), Museologia e Patrimônio (Museol Patr), Perspectivas em Gestão \& Conhecimento (Persp G\&C), PontodeAcesso (PdAcesso), Revista Brasileira de Biblioteconomia e Documentação (RBBD), Revista Digital de Biblioteconomia e Ciência da Informação (RDBCI), Revista Eletrônica de Comunicação, Informação \& Inovação em Saúde (RECIIS) e Revista Ibero-Americana de Ciência da Informação (RICI).
} 